\title{
Sciatic Nerve Block with Ropivacaine and Prilocaine in a Patient with Chronic Progressive External Ophthalmoplegia (CPEO)
}

\author{
Heiko Baumann*, Eduard Schlegel, Fabian Wagner, Jürgen Biscoping \\ Department of Anesthesia and Intensive Care, St. Vincentius-Kliniken gAG, Karlsruhe, Germany. \\ Email: *Heiko.Baumann@vincentius-ka.de
}

Received June $7^{\text {th }}$, 2012; revised July $8^{\text {th }}$, 2012; accepted August $5^{\text {th }}, 2012$

\begin{abstract}
The anesthetic implications of caring for patients with mitochondrial myopathies (MM) are not well defined, and the few case reports that have been published are contradictory. Local anesthetics particularly have the potential to worsen neuromuscular manifestations via direct effects on mitochondrial bioenergetics. Here we describe the anesthetic management of a patient with chronic progressive external ophthalmoplegia (CPEO) in which a sciatic nerve block with ropivacaine and prilocaine was performed and review the available related literature.
\end{abstract}

Keywords: Local Anesthetics; Mitochondrial Myopathy; Sciatic Nerve Block; Pharmacology; Anesthetic Management

\section{Introduction}

Mitochondrial toxicity by pharmacological compounds is a cause of myopathies and cardiomyopathies. Undesirable effects of several drugs including anticonvulsants, psychotropics, cholesterol medications, analgesics and anti-inflammatory pain relievers have been reported [1]. Local anesthetics exhibit a negative effect on mitochondrial energy metabolism and may cause severe adverse reactions in patients suffering from a mitochondrial myopathy. We describe the perioperative anesthetic management and pain management of a patient with chronic progressive external ophthalmoplegia scheduled for complex forefoot and ankle surgery. The use of ropivacaine in combination with prilocaine for peripheral nerve block in such a patient has not been previously reported.

\section{Case Report}

Informed consent was obtained to publish this case report. A 60-year-old woman with history of chronic progressive external ophthalmoplegia (CPEO) presented for a complex forefoot and ankle surgery. According to her medical history, she had no major acute or chronic illnesses. She had a known allergy to penicillin and nickel and class I obesity (body mass index: 32). CPEO was primarily diagnosed in 1990 by muscle biopsy and was reconfirmed in 1994 with no evidence of cerebral or cardiac

\footnotetext{
*Corresponding author.
}

manifestations (Friedrich-Baur-Institute, Ludwig Maximilians University Munich, Germany). At that time, the patient had complained of progressive bilateral ptosis and diplopic images. A myoadenylate deaminase (MAD) deficiency was ruled out by molecular genetic testing. Ptosis surgery was performed. Advancing difficulties in walking were reported. No history of cardiac dysrhythmia was reported. The patient had recently been taking Coenzyme $\mathrm{Q}_{10}$ and L-Carnitine. Preoperative routine laboratory parameters (full blood count, sodium, potassium, calcium, international normalized ratio [INR], creatinine, blood urea nitrogen [BUN], glomerular filtration rate [GFR], blood glucose level, c-reactive protein [CRP]) were in the normal range, and chest X-ray and electrocardography (ECG) showed no abnormalities.

We planned for total intravenous anesthesia (propofol, remifentanil) and sciatic nerve block for perioperative pain control. Flunitrazepam $(0.5 \mathrm{mg})$ was given as premedication. Pulse oximetry, continuous electrocardiogram, and arterial blood pressure were monitored. In an ultrasound-guided posterior approach in the lateral decubitus position combined with a stimulation technique (Stimuplex D., B. Braun, Germany), the distal sciatic nerve was identified and $400 \mathrm{mg}$ prilocaine $(20 \mathrm{ml} \mathrm{Xy}-$ lonest ${ }^{\circledR} 1 \%+10 \mathrm{ml}$ Xylonest ${ }^{\circledR} 2 \%$, AstraZeneca, Germany) and $150 \mathrm{mg}$ ropivacaine $\left(20 \mathrm{ml}\right.$ Naropin ${ }^{\circledR} 7.5$ $\mathrm{mg} / \mathrm{ml}$ AstraZeneca, Germany) were administered via a single shot technique. General anesthesia was induced with $130 \mathrm{mg}$ propofol after remifentanil infusion was 
started at $0.5 \mu \mathrm{g} / \mathrm{kg} / \mathrm{min}$. A laryngeal mask size 4 (LMA Supreme $^{\mathrm{TM}}$, LMA, Germany) was inserted to facilitate mechanical ventilation with a mixture of air and oxygen (50:50). Anesthesia was maintained with controlled infusion of propofol (3 - $5 \mathrm{mg} / \mathrm{kg} / \mathrm{h})$ and remifentanil (0.05 $0.1 \mu \mathrm{g} / \mathrm{kg} / \mathrm{min}$ ). No muscle relaxation was necessary. Arterial blood pressure, heart rate, percutaneous hemoglobin oxygen saturation, end-tidal carbon dioxide concentration, and body temperature remained stable throughout the procedure. The duration of surgery was $130 \mathrm{~min}$, and the propofol and remifentanil infusion were discontinued at the completion of skin suturing. The patient started breathing spontaneously and was awake $10 \mathrm{~min}$ later. The immediate pain scale was $<4$ on the numeric rating scale (NRS-11). The patient was continuously monitored in the anesthetic recovery room for an additional 10 hours. No supplemental analgesics were requested for the next 9.5 hours after the sciatic nerve block was performed. The blockade resolved completely, and there was no residual sensory or motoric deficiency after 11 hours post induction of anesthesia. Piritramide (Dipidolor ${ }^{\circledR}$, Janssen-Cilag, Germany) was used safely to treat further postoperative pain. The patient remained in the hospital for 12 days after surgery. The patient was doing well 2 months after surgery without any evidence of aggravation of the neuromuscular problems.

\section{Discussion}

The mitochondrial myopathies (MMs) consist of a heterogeneous group of disorders caused by structural and functional abnormalities in mitochondria leading to the involvement of the nervous system and muscles as well as other organ systems [2]. There might be an increased susceptibility for malignant hyperthermia (MH) [3]. On one hand, $\mathrm{MH}$ has been described in at least one case in a patient with MM [4]. On the other hand, there are MM cases in which patients received MH-triggering substances that did not lead to any complications [3,5]. Primum non nocere we planned a nontriggering anaesthetic technique with propofol and remifentanil infusions [6]. We chose a lactate-free, balanced crystalloid (Jonosteril ${ }^{\circledR}$, Fresenius Kabi, Germany) in order to avoid an iatrogenic lactate load due to the use of Lactated Ringer's solution [7]. Local anesthetic (LA) exhibits mitochondrial toxicity $[1,8]$, and thus, its safe application in patients with MM remains unclear. Bupivacaine, levobupivacaine, and ropivacaine decrease mitochondrial energy metabolism by uncoupling oxidative phosphorylation in mitochondria and enhance sarcoplasmic reticulum $\mathrm{Ca}^{2+}$ release [9]. They may further induce myotoxicity in isolated muscle fibers $[10,11]$. In one patient with MM, Maslow and Lisbon performed spinal anesthesia using hyperbaric tetracaine [12], a potent LA of the ester group. The pa- tient initially had decreased balance when walking and mild decreased sensation of the right foot. Finsterer reported a diffuse weakness, reduced tendon reflexes, absent patellar tendon reflexes, and subclonic achilles tendon reflexes as severe adverse reactions on infiltration of the submandibular nerve with the amide LA articaine in a patient with a respiratory chain disorder [13]. These findings could be the effect of the LA or may be of multifactorial cause. The disturbance of lactate or glucose levels resulting from defects in the respiratory chain necessitating anaerobic metabolism of glucose could deteriorate neuromuscular weakness. Furthermore, inadequate oxygen balance, unstable cardiovascular function and electrolyte shifts as a result of lactic acidosis are potential causes for the observed worsening neuromuscular manifestations in MM [14]. In our case, no neurologic deficit was noticed, and the sensibility and measurement of crude strength in arms and legs gained preoperative baseline parameters. Postoperative mobilization was possible in a timely manner. The interval to complete recovery from sciatic nerve block was in the typical time frame for the long-acting amid LA ropivacaine [15]. Using ropivacaine instead of bupivacaine seems to have advantages in patients with $\mathrm{MM}$ because mitochondrial energy metabolism and ATP synthesis are less inhibited by ropivacaine than by bupivacaine or levobupivacaine [16]. The lower lipid solubility of ropivacaine is thought to be responsible for the lesser dose-dependent effects on mitochondrial bioenergetics [17].

\section{Conclusion}

This uneventful recovery from distal sciatic nerve block with no observed side effects might provide the first evidence that the use of prilocaine and ropivacaine at standard clinical dosages for peripheral nerve block is safe in patients with CPEO. As reported before, total intravenous anesthesia (propofol, remifentanil) is a safe choice for these patients.

\section{REFERENCES}

[1] K. Nouette-Gaulain, C. Jose, X. Capdevila and R. Rossignol, "From Analgesia to Myopathy: When Local Anesthetics Impair the Mitochondrion,” The International Journal of Biochemistry \& Cell Biology, Vol. 43, No. 1, 2011, pp. 14-19. doi:10.1016/j.biocel.2010.10.005

[2] E. A. Shipton and D. O. Prosser, "Mitochondrial Myopathies and Anaesthesia," European Journal of Anaesthesiology, Vol. 21, 2004, pp. 173-178.

[3] R. M. Fricker, T. Raffelsberger, S. Rauch-Shorny, J. Finsterer, C. Muller-Reible, H. Gilly and R. E. Bittner, "Positive Malignant Hyperthermia Susceptibility in Vitro Test in a Patient with Mitochondrial Myopathy and Myoadenylate Deaminase Deficiency,” Anesthesiology, 
Vol. 97, No. 6, 2002, pp. 1635-1637. doi:10.1097/00000542-200212000-00044

[4] Y. Ohtani, T. Miike and T. Ishitsu, "A Case of Malignant Hyperthermia with Mitochondrial Dysfunction (Letter)," Brain \& Development, Vol. 7, 1985, p. 249.

[5] A. M. Burns and M. P. Shelly, "Anaesthesia for Patients with Mitochondrial Myopathy,” Anaesthesia, Vol. 44, No. 12, 1989, pp. 975-977. doi:10.1111/j.1365-2044.1989.tb09200.x

[6] E. Guasch, B. Civantos, J. M. Aguilar, M. D. Torres and F. Gilsanz, "Progressive External Ophthalmoplegia and Ambulatory Remifentanil-Propofol Based Anaesthesia,” Anaesthesia, Vol. 58, No. 6, 2003, pp. 607-608. doi:10.1046/j.1365-2044.2003.32191.x

[7] S. Muravchick and R. J. Levy, "Clinical Implications of Mitochondrial Dysfunction,” Anesthesiology, Vol. 105, No. 4, 2006, pp. 819-837. doi:10.1097/00000542-200610000-00029

[8] V. A. Sardao, S. L. Pereira and P. J. Oliveira, "DrugInduced Mitochondrial Dysfunction in Cardiac and Skeletal Muscle Injury,” Expert Opinion on Drug Safety, Vol. 7, No. 2, 2008, pp. 129-146. doi:10.1517/14740338.7.2.129

[9] K. Nouette-Gaulain, P. Sirvent, M. Canal-Raffin, D. Morau, M. Malgat, M. Molimard, J. Mercier, A. Lacampagne, F. Sztark and X. Capdevila, "Effects of Intermittent Femoral Nerve Injections of Bupivacaine, Levobupivacaine, and Ropivacaine on Mitochondrial Energy Metabolism and Intracellular Calcium Homeostasis in Rat Psoas Muscle,” Anesthesiology, Vol. 106, No. 5, 2007, pp. 1026-1034. doi:10.1097/01.anes.0000265164.29630.b4

[10] I. W. rwin, E. Fontaine, L. Agnolucci, D. Penzo, R. Betto, S. Bortolotto, C. Reggiani, G. Salviati and P. Bernardi, "Bupivacaine Myotoxicity Is Mediated by Mitochondria," The Journal of Biological Chemistry, Vol. 277, No. 14,
2002, pp. 12221-12227. doi:10.1074/jbc.M108938200

[11] Q. Hogan, R. Dotson, S. Erickson, R. Kettler, K. Hogan, "Local Anesthetic Myotoxicity: A Case and Review," Anesthesiology, Vol. 80, No. 4, 1994, pp. 942-927. doi:10.1097/00000542-199404000-00029

[12] A. Maslow and A. Lisbon, "Anesthetic Considerations in Patients with Mitochondrial Dysfunction,” Anesthesia \& Analgesia, Vol. 76, No. 4, 1993, pp. 884-886. doi:10.1213/00000539-199304000-00035

[13] J. Finsterer, C. Haberler and J. Schmiedel, “Deterioration of Kearns-Sayre Syndrome Following Articaine Administration for Local Anesthesia," Clinical Neuropharmacology, Vol. 28, No. 5, 2005, pp. 148-149. doi:10.1097/01.wnf.0000165352.10841.21

[14] V. A. Thompson and J. A. Wahr, "Anesthetic Considerations in Patients Presenting with Mitochondrial Myopathy, Encephalopathy, Lactic Acidosis, and Stroke-Like Episodes (MELAS) Syndrome,” Anesthesia \& Analgesia, Vol. 85, No. 6, 1997, pp. 1404-1406.

[15] V. Domingo-Triado, S. Selfa, F. Martinez, D. SanchezContreras, M. Reche, J. Tecles, M. T. Crespo, J. M. Palanca and B. Moro, "Ultrasound Guidance for Lateral Midfemoral Sciatic Nerve Block: A Prospective, Comparative, Randomized Study," Anesthesia \& Analgesia, Vol. 104, No. 5, 2007, pp. 1270-1274.

[16] F. Sztark, M. Malgat, P. Dabadie and J. P. Mazat, "Comparison of the Effects of Bupivacaine and Ropivacaine on Heart Cell Mitochondrial Bioenergetics,” Anesthesiology, Vol. 88, No. 5, 1998, pp. 1340-1349. doi:10.1097/00000542-199805000-00026

[17] F. Sztark, K. Nouette-Gaulain, M. Malgat, P. Dabadie and J. P. Mazat, "Absence of Stereospecific Effects of Bupivacaine Isomers on Heart Mitochondrial Bioenergetics," Anesthesiology, Vol. 93, No. 2, 2000, pp. 456-462. doi:10.1097/00000542-200008000-00025 